\title{
Simultaneous pollutant removal and PHB accumulation in simple anaerobic treatment of oily wastewater
}

\author{
Budhi Primasari ${ }^{1 *}$ and Tong Koh Wei ${ }^{2}$ \\ ${ }^{1}$ Department of Environmental Engineering, Universitas Andalas, Padang, Indonesia \\ ${ }^{2}$ Department of Environmental Engineering, University of Malaya, Kuala Lumpur, Malaysia
}

\begin{abstract}
Oil and grease is one of the complicated pollutants to be removed due to oil-in-water emulsions content. This research is a treatment of oily wastewater from an edible oil factory. Addition to pollutant removal, accumulation of PHB (Polyhydroxybutyrate) is investigated simultaneously. Objectives of this research are to obtain the optimum operating conditions for removal of COD and oil, grease, and accumulation of PHB. Operating parameters are acclimatization period (3-7 days), $\mathrm{pH}$ (6-8) and sludge concentration (25-75\%). Anaerobic treatment was conducted in a bottle and put on a shaker. The wastewater from the edible oil factory was fed to reactors as the substrate and the sludge was added. The result shows that this treatment removed $59.4-90.4 \%$ of COD and $65.3-85.2 \%$ of oil and grease. Overall PHB accumulation was $0.67-6.00 \%$ of PHB/VSS, which is much lower than the pure culture. The influence of $\mathrm{pH}(6-8)$ is less significant to both pollutant removal and PHB accumulation. The COD removal efficiency increased with the period of acclimatization and 5 days of acclimatization shows the highest yield of PHB. 50\% sludge concentration shows the highest yield of PHB, which is $6.0 \% \mathrm{PHB} / \mathrm{VSS}$.
\end{abstract}

\section{Introduction}

Oil and grease are one amongst complicated pollutant to remove, due to its characteristics, oil-water emulsions. These emulsified oil droplets are sheltered from spontaneous coalescence into larger flocs, thus making oil separation by simple gravity a timeconsuming and challenging process. Due to the enormous scale of this industry, the palm oil-related effluents have been increasing at an alarming rate, and the effects are detrimental to both the environment and the human being [1]. To treat oil and grease rich wastewaters biologically the most important criteria to be met are stable and active biomass and proper sludge concentration. Hence, this paper is aimed at achieving the goal by using a simple anaerobic batch reactor to treat the oily containing wastewater through assessing the effects of various sludge concentrations, $\mathrm{pH}$ variation, and acclimatization periods on the oily effluent quality. Also, due to the nature of the wastewater, the microbial activity throughout

\footnotetext{
*Corresponding author: budhiprimasari@eng.unand.ac.id
} 
the treatment will result in the accumulation of biodegradable polyester, Polyhydroxybutyrate (PHB) which can be used in the manufacturing of biodegradable plastics. In short, the proposed reactor will not only reduce the pollutant in the effluents, but the end products of this reaction will produce valuable material [2].

The overall aim of this study is to examine the performance of the simple anaerobic batch reactor regarding wastewater removal efficiency for refined palm oil effluent treatment. The specific objectives are to investigate the feasibility and effectiveness of simple anaerobic batch reactor to treat oily containing wastewater. Also, the effects of sludge concentration, acclimatization periods, and $\mathrm{pH}$ variation on the quality of effluent and to the accumulation of Polyhydroxybutyrate (PHB) were also determined.

Biodegradable plastics are polymer species. Biodegradable plastics that are compostable can be treated biologically together with other bio-waste. Plastic bags are generally made from non-renewable petroleum resources. In general, biodegradable plastics whose components are derived from renewable raw materials can be made from abundant agricultural/ animal resources like cellulose, starch, collagen, casein, soy protein polyesters are triglycerides. Biodegradable plastics degrade over a period if exposed to sun and air. Use of plastics in agriculture and rural communities has extensively increased [3]. The increasing use of polymers for disposable items such as packaging creates problems for garbage disposal and has produced a demand for biodegradable polymers [3]. Biodegradability of plastics has been proposed as a solution to the waste problem. Biodegradable plastics have an expanding range of potential applications, and they are environmentally friendly. Therefore, there is a growing interest in degradable plastics, which degrade more rapidly than conventional disposable.

The biodegradability of plastics is dependent on the chemical structure of the material and the constitution of the final product. Therefore, biodegradable plastics can be based on natural or synthetic resins. Natural biodegradable plastics are based primarily on renewable resources and can be either naturally produced or synthesized from renewable resources. Biodegradation is degradation caused by biological activity, particularly by enzyme action leading to significant changes in the materials chemical structure. Non-renewable synthetic biodegradable plastics are petroleum-based. As any marketable plastic product must meet the performance requirements of its intended function, many natural biodegradable plastics are blended with synthetic polymers to produce plastics that meet these functional requirements.

\section{Materials and Methods}

The main equipment used for this study is the serum bottle which has been adopted as the simple batch reactor due to its simplicity and size. The volume of this serum bottle is 125 $\mathrm{ml}$ which are suitable for this study as the volume for the treatment has been set for $100 \mathrm{ml}$ for each sample. However, slight adjustment such as a more secure seal has been used to make sure the suitability of the serum bottle to be used as the simple anaerobic batch reactor.

The mechanical shaker has multiple settings that can be used based on user requirements such as shaker rotational speeds, temperature, and timer of usage. The capacity of this shaker can fit up to about 20 serum bottles at a time, and the most significant volume of apparatus that can be fitted in is $250 \mathrm{ml}$ conical flask. Also, this shaker has tinted glass which minimizes the light penetration which is useful for the anaerobic condition. Also, all of this equipment has been tested before the actual experimental runs to achieve the constant and satisfactory state.

The relevant pollutant parameters of the effluent from a palm oil refinery factory are based on their physical and chemical properties, which include temperature, $\mathrm{pH}$, Chemical 
Oxygen Demand (COD), Total Suspended Solids (TSS), Volatile Suspended Solids (VSS), and Oil and Grease. Analyses of COD, TSS, VSS, Oil, and Grease referred to APHA Standard Method 5220 D, 2540D, 2540e, 5220B. Studies of PHB Concentration followed the Assay method proposed by Law Slepecky [4]. These parameters were determined to obtain the wastewater characteristics. The sequencing batch reactor sludge was taken from the sequencing batch reactor at the same palm oil refinery factory. The oily effluent as the food for the biomass was fed only once into the reactor which seeded with sludge as this is not a continuous fed-batch reactor.

The serum bottles were secured correctly and placed in the mechanical shaker during the acclimatization process to ensure an anaerobic environment and to maintain the sludge in well-mixed condition with the oily effluent. The oily wastewater from the edible oil refinery factory was used as the feed. The wastewater was feed into the reactor according to the ratio set before the experiment. In general, there are 3 main variables have been established for this experiment which are acclimatization periods $(3,5$, and 7 days of acclimatization), $\mathrm{pH}$ range from 6 to 8 , and lastly sludge concentration in the reactor $(25 \%$, $50 \%$, and $75 \%$ of sludge concentration) which in favour of PHB accumulation (Qu et al, 2009; Chua et al, 2003).

\section{Results and discussion}

\subsection{COD and O\&G removal percentage}

The 3, 5, and 7 days of acclimatization periods have been set as variables in determining the optimum conditions for treatment of oily effluent using simple anaerobic batch reactor. The COD removal percentages ranged from 59.38-90.63\% which shown in Fig. 1 . From the Fig. 1, it has been demonstrated that as acclimatization period increases, the COD removal strength of the reactor also increases slightly. On average, the 7 days of acclimatization was observed to have marginally better COD removal efficiency than those samples of 3 and 5 acclimatization days. This might due to the biomass has longer retention time in the reactor.

As for the O\&G removal strength, the results (Fig. 2) have shown that on average [5], shorter acclimatization period has slightly higher removal strength which might due to the removal mechanism of O\&G is faster than that of COD removal. However, the reduction in $\mathrm{O} \& \mathrm{G}$ removal strength as acclimatization period increases might indicate that the O\&G removal mechanism is less sensitive in more extended acclimatization period [6].

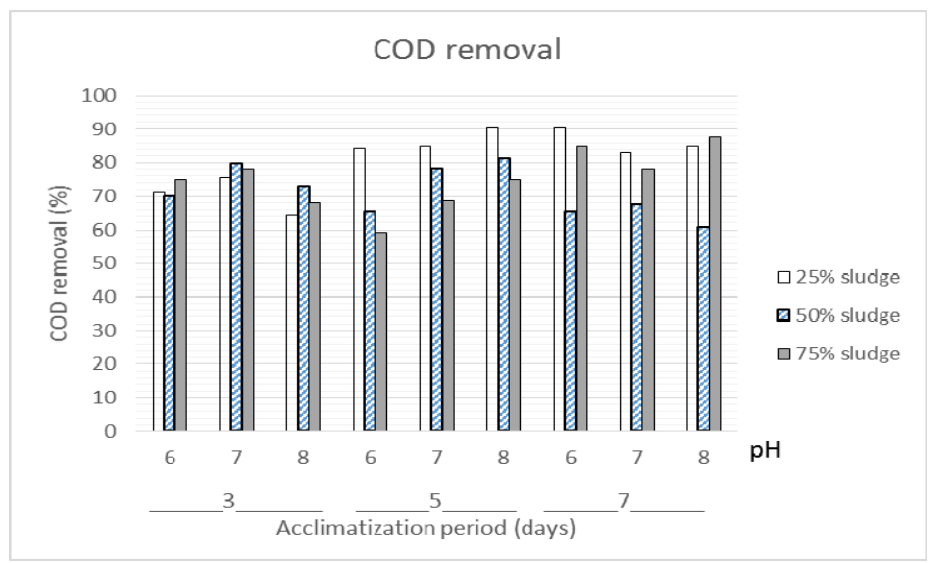

Fig. 1. The effect to COD removal. 


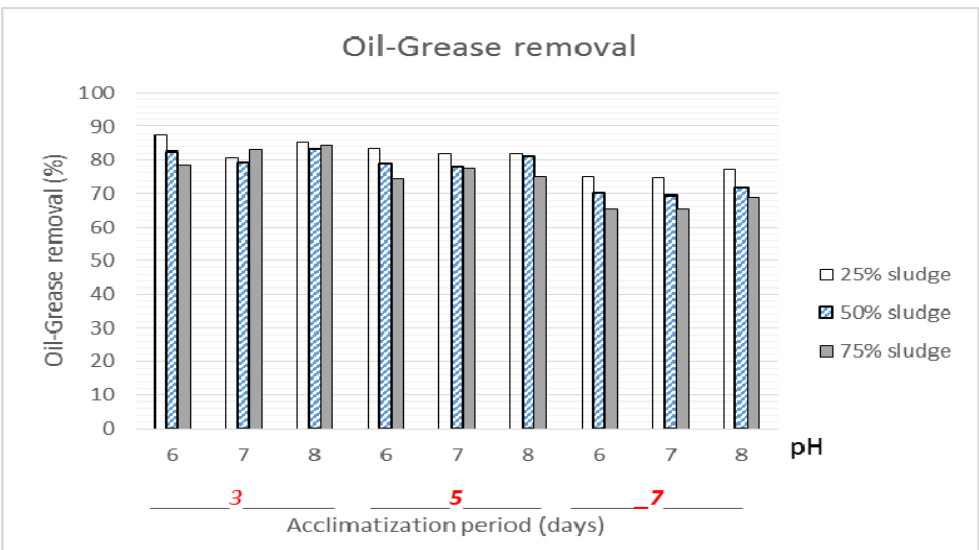

Fig. 2. The effect to oil and grease removal.

\subsection{Accumulation and yield of PHB}

With the sludge acclimatized in the simple anaerobic batch reactors, there are a total of 3 main batches of an experiment which comprise of 3, 5, and 7 days of acclimatization for accumulations and yield of PHB were conducted. From Fig. 3 and Fig. 4, the research of 5 days acclimatization shows higher PHB accumulation and yield than the rest with lowest PHB accumulation and yield was observed in the samples of 3 days of acclimatization. In general, the yield of PHB ranged from $0.67 \%$ to $6.0 \%$ of PHB/VSS [2].

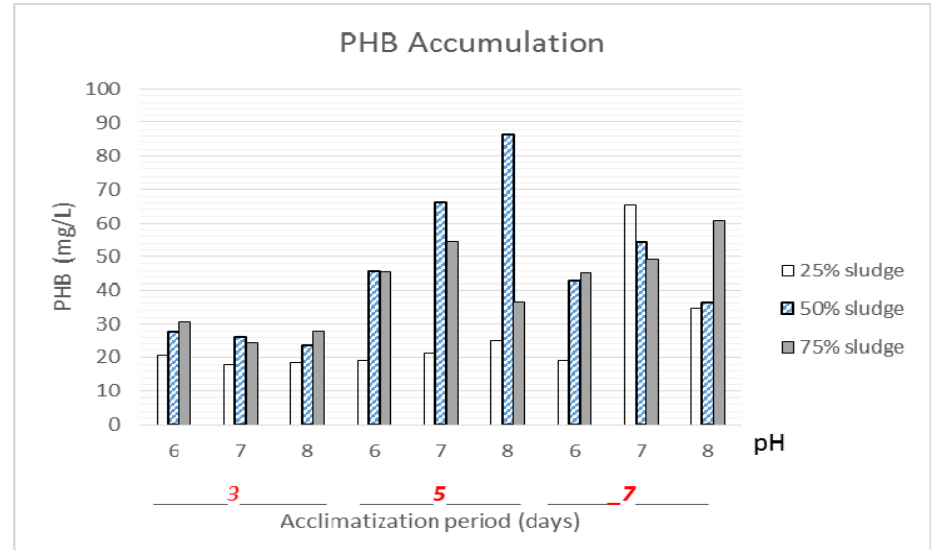

Fig. 3. The effect to PHB accumulation.

The reason for such a trend is unknown. However, there are some possible reasons to explain the variation of the yield and accumulation of PHB caused by the difference in the acclimatization period. Firstly, the sludge acclimatization period theoretically determines to mean microbial life-time and hence microbial population. From the results, the 5 days acclimatization may select microbial community with more significant PHB production capacity than that selected under longer or shorter acclimatization period. Also, it could be due to the differences in acclimatization periods which might have affected the PHB accumulation capability of sludge via the difference in the organic loading to biomass. Also, the more extended acclimatization period might contain a higher amount of inert biomass, and this could contribute to lower PHB accumulation in samples of 7 days of 
acclimatization. Hence, it was evident that the acclimatization period could significantly influence the PHB accumulation.

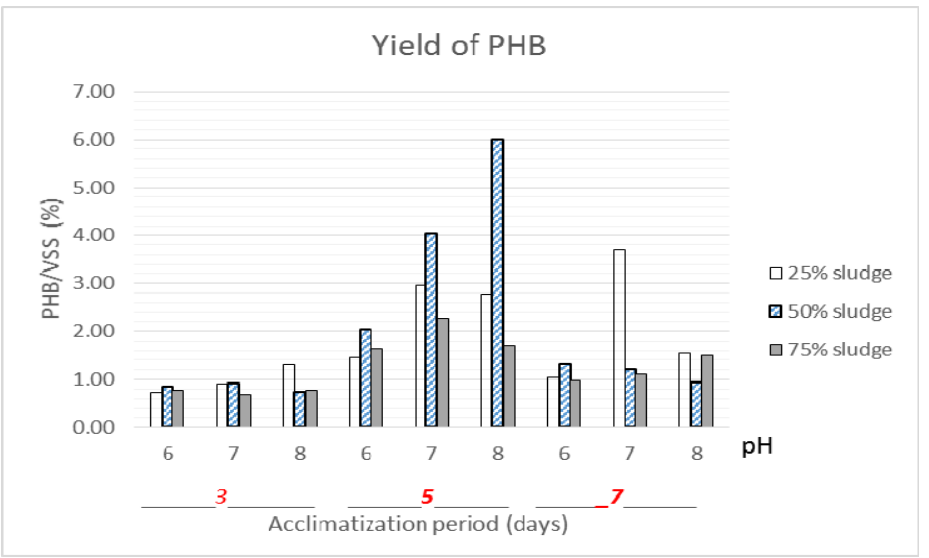

Fig. 4. The effect to yield of PHB.

\subsection{The growth of biomass}

Based on Fig. 5, the growth of biomass in the samples can be observed in all samples after treatment. Furthermore, it was found that at longer acclimatization period, the growth of biomass was higher compared with the rest. This is due to as the acclimatization period increases, the higher the biomass concentration in the reactor. Due to the difference in the acclimatization period, microorganisms in longer acclimatization had a better chance of taking up more organic substrates than those in shorter acclimatization period. This might have led to the higher PHB accumulation capability of sludge for longer acclimatization period.

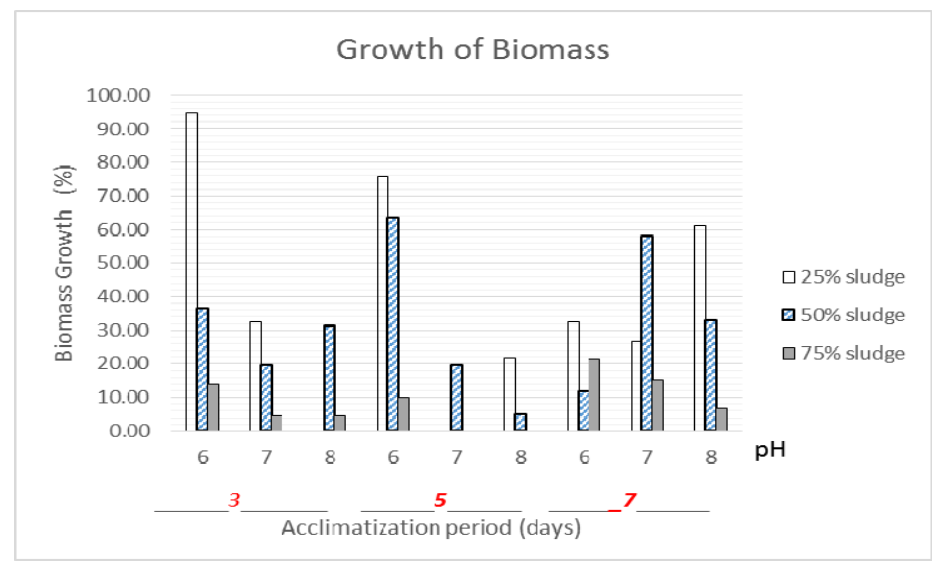

Fig. 5. The effect to biomass growth.

\section{Conclusions}

It was found that the acclimatization periods have a significant effect on the COD removal efficiency, accumulation, yield of PHB and growth of biomass. As the acclimatization period increases, the COD removal efficiency is higher, but oil and grease removal strength 
showed a slight decreasing. Also, it was observed that samples 5 days of acclimatization have better accumulation and yield of PHB. However, further studies should intensify the research on acclimatization period by focusing on the range of 3 to 5 days of acclimatization.

Sludge concentration is rather crucial as this is a biological treatment. Proper F/M ratio is needed to achieve optimum pollutants removal. In this study, it was found that samples with $25 \%$ sludge concentration have slightly better removal efficiency whereas samples with 50\% sludge concentration have better yield and accumulation of PHB. The growth of biomass is more favorable in samples with $25 \%$ sludge concentration which might due to the ample supply of substrate for the microorganism's growth. Hence, future studies are essential to further evaluate the effect of sludge concentrations by focusing the range which closes to $50 \%$ sludge concentration.

One of the objectives of this research is to study the PHB production system in which excess sludge of the industrial effluent treatment process was utilized as PHB production of bacterial cultures. Also, this research was aimed to investigate the optimum operating conditions of the sludge process for enhancing the PHB [7] production capability of sludge. Although the PHB content ranged from 0.67-6.00\% PHB/VSS is much lower than pure culture [8], but this proposed method could be considered as an environment-friendly means to convert waste into a valuable product.

\section{References}

1. S. Indah, D. Helard, A. Sasmita, Water Sci. Technol. 73, 12 (2016)

2. S.R. Pandian, V. Deepak, K. Kalishwaralal, N. Rameshkumar, M. Jeyaraj, S. Gurunathan, Bioresour. Technol. 101, 2 (2010)

3. J.-W. Rhim, P.K.W.Ng, Crit. Rev. Food Sci. Nutr. 47, 4 (2007)

4. J.H. Law, R.A. Slepecky, Ralph a. slepecky (1960)

5. G. Du, J. Chen, H. Gao, Y. Chen, S. Lun, Microbiol. Rev. (2000)

6. F. Ji, C. Li, X. Dong, Y. Li, D. Wang, J. Hazard. Mater. 164, 2-3 (2009)

7. D. Fernandez, E. Rodriguez, M. Bassas, M. Vinas, A.M. Solanas, J. Llorens, A.M. Marques, A. Manresa. Biochem. Eng. J. 26, 2-3 (2005)

8. M. Majone, M. Beccari, D. Dionisi, C. Levantesi, R. Ramadori, V. Tandoi, Water Res. 41, 1 (2007) 